\title{
Mechanismenentwicklung eines Assistenzsystems für die oberen Extremitäten
}

\author{
Daniel Garbe ${ }^{1}$, Maik Berger ${ }^{2}$ \\ 1 daniel.garbe@mb.tu-chemnitz.de \\ 2 maik.berger@mb.tu-chemnitz.de \\ 1,2 Professur Montage- und Handhabungstechnik, \\ Technische Universität Chemnitz, Deutschland
}

DOI: 10.14464/awic.v3i0.397

\section{ABSTRACT}

In diesem Beitrag werden mobile Assistenzsysteme, sog. Exoskelette, mit ihren charakteristischen Eigenschaften vorgestellt. Schwerpunkte bilden die Entwicklung der Mechanik für die Nachbildung der menschlichen Bewegung und das Antriebssystem. Auf Grundlage der menschlichen Anatomie, mit Bezug zum Schultergürtel, Schultergelenk und Ellenbogengelenk, wird ein Mechanismus entwickelt, der die Armbewegung beim Heben, Halten und Tragen von Lasten nachbilden kann. Weiterführend wird die Entwicklung eines aktiven Antriebssystems für die Unterstützung der zuvor genannten Aufgaben beschrieben. Für die Auslegung werden Richtwerte der Bundesanstalt für Arbeitsschutz und Arbeitsmedizin (BAuA) herangezogen. Der Einsatz pneumatischer Muskeln als Aktuator bringt Vorteile hinsichtlich eines hohen spezifischen Arbeitsvermögens mit sich. Ein Nachteil ist jedoch die charakteristisch nichtlineare Kraft-Kontraktionskennlinie. Es folgt eine Beschreibung für die ungleichmäßige Übersetzung pneumatischer Muskeln mit einem Bandmechanismus. Die verwendete Methode gestattet nicht nur eine Kompensation der nachteiligen, degressiven Kraftkennlinie. Sie erlaubt darüber hinaus die anwendungsspezifische Veränderung des Arbeitskennfeldes. Abschließend erfolgt die Überführung des Bandmechanismus in eine zweistufige Übersetzung, welche die Voraussetzungen pneumatischer Muskeln für den Einsatz in mobilen Anwendungen verbessert.

Keywords: Exoskelett, pneumatischer Muskel, Bandmechanismen, ungleichmäßige Übersetzung 


\section{EINFÜHRUNG}

Tragbare Assistenzsysteme für die Unterstützung der menschlichen Bewegung werden heute gedanklich meist mit Sciencefiction verbunden, obwohl die Forschung in diesem Bereich schon seit über 50 Jahren tätig ist. Nach Looze, Bosch und Krause (2016) steht die Etablierung dieser Systeme noch am Anfang und begrenzt sich auf Speziallösungen, von denen viele bisher nur im Labor getestet wurden. Gründe dafür sind wahrscheinlich der hohe Spezialisierungsgrad der einzelnen Entwicklungen und die unzureichende Ergonomie beim Tragen. Die technische Unterstützung des Anwenders in Zusammenhang mit der Tragbarkeit sind wichtige Kriterien die im weiteren Beitrag behandelt werden.

Die häufigsten Aufgaben, die ein Exoskelett für die oberen Extremitäten im industriellen Umfeld erfüllen muss, sind das Heben, Halten und Tragen von Lasten. In den letzten Jahren sind verstärkt Entwicklungen zu aktiven und passiven Assistenzsystemen begonnen worden. Ziel ist die Entlastung des Anwenders bei seiner Arbeit, ohne inn in seiner Beweglichkeit einzuschränken. Herr (2009) beschreibt, dass Passive Systeme potentielle Energie aus Speichern wie bspw. Federn nutzen und damit die Gewichtskraft der Gliedaßen mit einer festgelegten Kraftkennlinie kompensieren. Im Gegensatz dazu arbeiten aktive Systeme mit verstellbaren Antrieben, deren gesamtes Arbeitsfeld für die Unterstützung flexibel genutzt werden kann. Die Lastabhängige Unterstützung der aktiven Systeme ist ein klarer Vorteil gegenüber den passiven Systemen. Abbildung 1 zeigt zwei aktiv unterstützende Exoskelette aus aktuellen Entwicklungen. Der Mechanismus der Stuttgart Exo-Jacket, wie er von Ebrahimi, Gröninger und Singer (2017) beschrieben wird, hat einen Armfreiheitsgrad von 6 und nutzt elektromechanische Antriebe an den Gelenken, deren Drehmoment über ein hoch übersetzendes Gleitkeilgetriebe gesteigert wird. Der Vorteil der hohen Kraftunterstützung geht mit einer hohen Steifigkeit des Antriebssystems einher, die den Anwender in seiner Selbstbestimmung eingrenzt. Eine Relativbewegung der angetriebenen Gelenke kann nur mittels geeigneter Sensorik und hohem steuerungs- und regelungstechnischem Aufwand gelöst werden. Im Vergleich dazu besitzt der Mechanismus des Muscle Suit, nach Murumatsu und Kobayashi (2014), einen Armfreiheitsgrad von 5 und verwendet pneumatische Muskeln als Antrieb. Die Kraftleitung erfolgt vom Rücken, über Seilzüge zu den einzelnen Gelenken und ermöglicht eine dezentrale Anbringung des Antriebes. Plettenburg (2005) beschreibt das hohe spezifisches Arbeitsvermögen, im Vergleich zu Pneumatikzylindern, was ein Vorteil für den Einsatz in mobilen Systemen ist. Der entscheidende Nachteil besteht jedoch in der nichtlinearen Kraft-Kontraktions-Kennlinie, bei der die Maximalkraft über die Kontraktion progressiv abfällt (Hesse, 2003). 

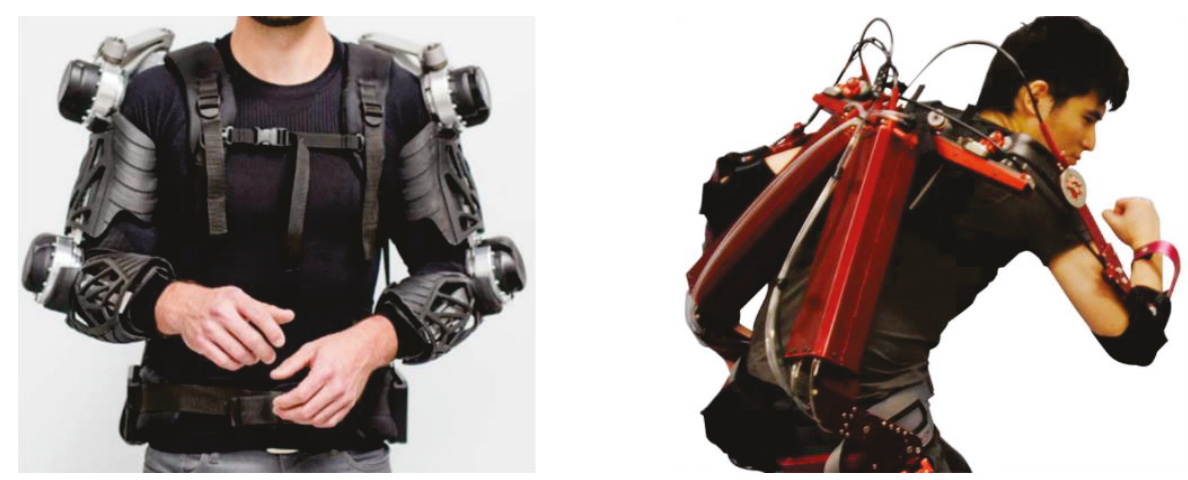

Abbildung 1: Exoskelette: links: Stuttgart Exo-Jacket; rechts: Muscle Suit

Quelle:

Ebrahimi et al. (2017); Murumatsu und Kobayashi (2014)

Die Nachteile der dargestellten Exoskelette verdeutlichen den Handlungsbedarf, vor allem im Bereich des Antriebssystems, aktiv unterstützender Assistenzsysteme. Die Bereitstellung einer ausreichend hohen Kraftunterstützung, bei gleichzeitiger Minimierung der Bewegungseinschränkung des Menschen sind die Ziele der folgenden Entwicklung.

\section{ANFORDERUNGEN AN DIE MECHANIK DES EXOSKELETTS}

Dieser Beitrag ist auf die Entwicklung der mechanischen Struktur zur Bewegungsnachbildung des Arms und des Antriebssystems für die Hebeunterstützung beschränkt. Die Anforderungen ergeben sich aus der notwendigen Bewegungsfreiheit des Menschen, der Anzahl aktuierter Gelenke und dem notwendigen Unterstützungsmoment, die im folgenden Abschnitt, in der angegebenen Reihenfolge beschrieben werden.

Für das Exoskelett ist eine uneingeschränkte Bewegung der Arme mit 150 Grad Flexion, 90 Grad Abduktion, 130/ 30 Grad Ante-/ Retroversion und 90/ 60 Grad Innen-/ Außenrotation, in Bezug auf die anatomische Normalstellung gefordert. Die Entwicklung des Bewegungsmechanismus kann mit zwei unterschiedliche Strategien erfolgen. Bei der Ersten kopiert das Exoskelett die menschlichen Gelenke, die auf mechanische Grundbewegungen reduziert werden (Palastanga, Soames und Eggers, 2015; Schünke, 2014). Die Schulter gleicht einem Kugel- und der Ellenbogen einem Scharniergelenk. Dieser Sachverhalt ist in Abbildung 2 dargestellt. 


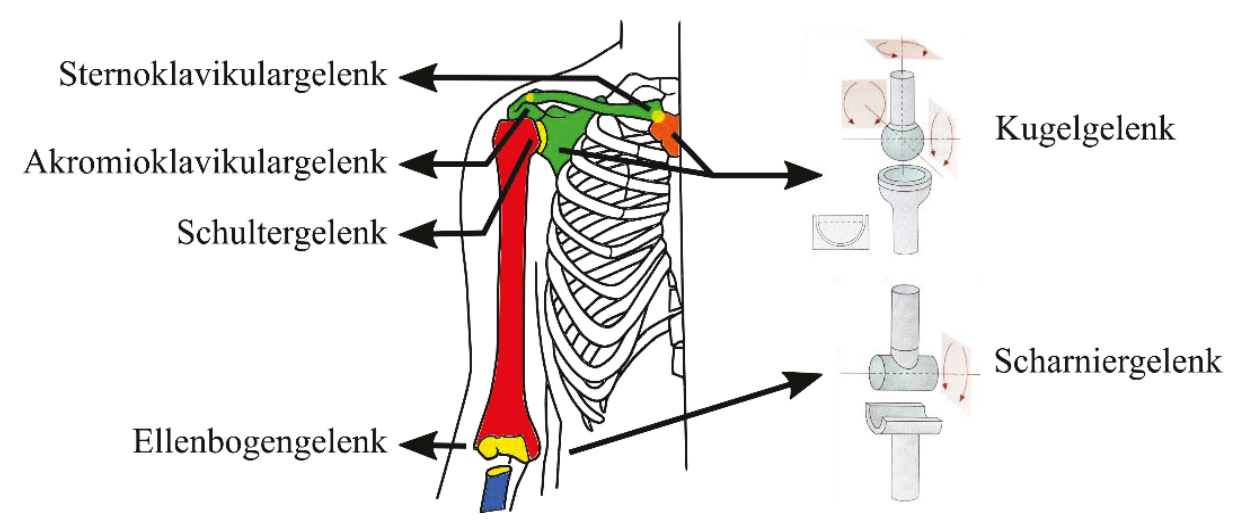

Abbildung 2: Obere Extremitäten und deren vereinfachte mechanische Gelenke Quelle: nach Palastanga et al. (2015); Schünke (2014)

Nach Tillmann (2017) und Palastanga et al. (2015) setzt sich die Oberarmbewegung aus mehreren Teilgelenken zusammen. Eine ausschließlich auf das Schultergelenk begrenzte Bewegung ist bis näherungsweise 90 Grad Anteversion, 25 Grad Abduktion bei gestrecktem Arm und 70/ 60 Grad Innen-/ Außenrotation möglich. Die Bewegungsfreiheit des Schultergelenks ist für die geplante Anwendung unzureichend und erfordert die Einbeziehung des Schultergürtels in die Gesamtbewegung. Dieser setzt sich aus Scapula, Clavicula und den zugehörigen Sternoklavikular- und Akromioklavikulargelenk zusammen (vgl. Abbildung 2). Bei vereinfachter Betrachtung handelt es sich beim Sternoklavikulargelenk ebenfalls um ein Kugelgelenk. Die zweite Strategie für die Imitation der Armbewegung zielt darauf ab, einen Ersatzmechanismus für die natürliche Bewegung zu finden. Eine Übereinstimmung der anatomischen und mechanischen Gelenke ist dabei nicht erforderlich. Nachteilig ist der meist höhere Freiheitsgrad.

Die Auswahl der aktiv unterstützenden Gelenke sind an den Richtlinien der BAuA ausgerichtet (Bundesanstalt für Arbeitsschutz und Arbeitsmedizin, 2001). Exoskelette für die oberen Extremitäten sollen vorrangig das Heben, Halten und Tragen erleichtern. Die Lasten sind vor dem Körper und ohne Verdrehung des Oberkörpers zu handhaben. Unter Beachtung der Leitmerkmalmethode treten die aus der Gewichtskraft resultierenden Belastungen vorrangig im Ellenbogen- und Schultergelenk, bei der Flexion/ Extension und Anteversion/ Retroversion auf. Adduktion/ Abduktion und Innen-/ Außenrotation der Schulter sind wegen der vergleichsweise geringen Gelenkmomente als passive Bewegungen zu betrachten. Für die Unterstützung einer allgemeinen Bewegung sind somit zwei angetriebene Gelenke je Arm erforderlich.

Bei der Antriebsauslegung ist das Unterstützungsmoment, in Abhängigkeit des Gelenkwinkels, maßgeblich. Ziel ist die Kompensation der durch die Gewichtskraft hervorgerufenen Gelenkmomente. Das Drehmoment ergibt sich aus dem Produkt des resultierenden Schwerpunktes aller Massen und dem wirkenden Hebelarm zum Drehzentrum der Gelenke. Nach Angaben von Steinberg und Windberg (2008) ist eine risikoverringerte Belastung, durch 
Zusatzgewichte für Männer, auf 7,5 kg je Arm begrenzt. Die maximalen Gelenkmomente betragen bei $15 \mathrm{~kg}$ zusätzlicher Gesamtlast (7,5 kg je Arm) 28,4 Nm im Ellenbogen und $54,7 \mathrm{Nm}$ in der Schulter, die vom Assistenzsystem, bei vollständiger Entlastung, aufgebracht werden müssen.

\section{MECHANISCHE ENTWICKLUNG DES ASSISTENZSYSTEMS}

\subsection{BEWEGUNGSMECHANISMUS}

Der Einsatzzweck des neuen Assistenzsystems umfasst die Unterstützung der oberen Extremitäten beim Heben, Halten und Tragen von Lasten. Die mit der Mechanik nachzubildende Bewegung des Arms ist durch die erforderliche Unterstützung festgelegt und schließt den Schultergürtel, die Schulter und den Ellenbogen ein. Eine Nachbildung des Schulter- und Ellenbogengelenks, durch die Vereinfachung auf mechanische Grundgelenke, ist zweckmäßig. Somit wird der Arm, nach dem Vorbild der menschlichen Anatomie, kopiert. Die Entwicklung eines Ersatzmechanismus ist hierfür nicht erforderlich. Abbildung 3 zeigt den vereinfachten Mechanismus mit Gliedern und Gelenkachsen. Für die theoretische Größenanpassung der Mechanik an den Menschen wurde das Menschmodell Manikin Lite der Firma PTC verwendet (Heine, 2010).

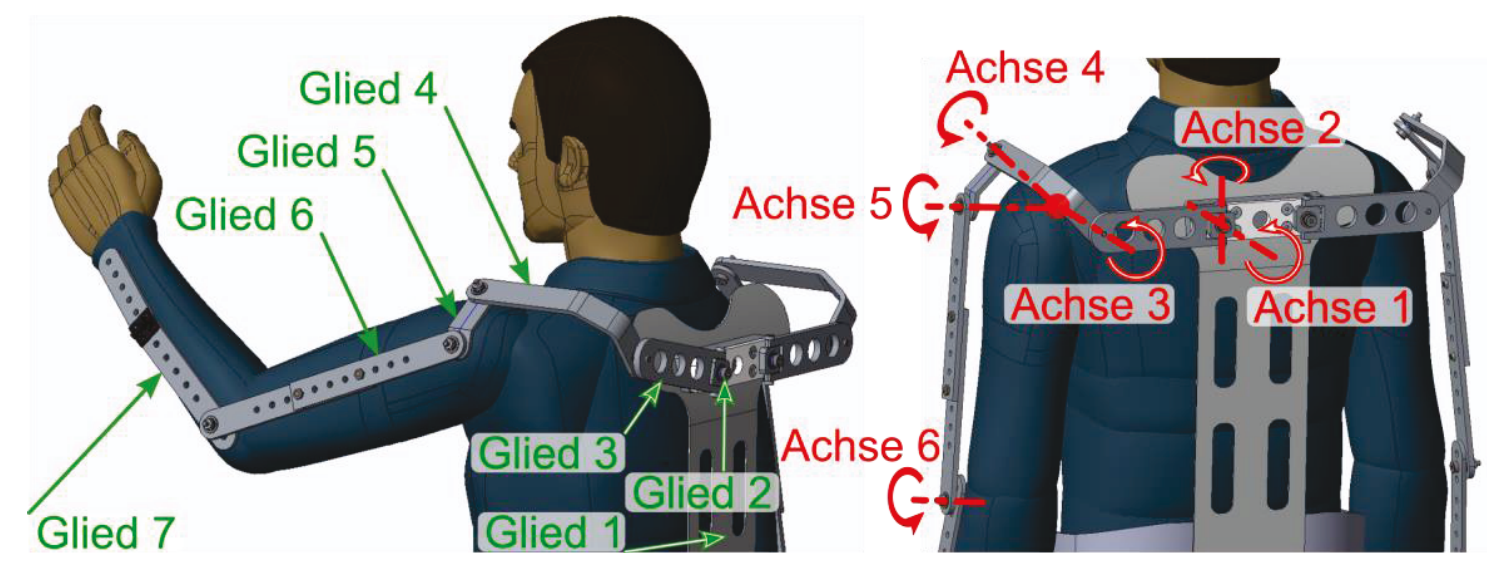

Abbildung 3: Menschmodell mit Exoskelett bei abduziertem Oberarm und flektiertem Unterarm Quelle: Eigene Darstellung

Das vereinfachte Scharniergelenk des Ellenbogens wird als Drehgelenk gestaltet. Der natürliche Bewegungsbereich der Flexion von 150 Grad ist mit dieser Ausführung ungehindert möglich. Das Kugelgelenk der Schulter wird mit drei Drehgelenken nachgebildet, deren Achsen sich in einem gemeinsamen Punkt, dem realen Drehzentrum der Schulter, schneiden. Bei einer senkrechten Ausrichtung der Achsen zueinander würde Glied 4 bei ca. 45 Grad Adduktion mit dem Nacken kollidieren. Mit einer veränderten Ausgangsposition von Glied 4, die mit einer Verringerung des Achswinkels zwischen Achse 4 und 5 einhergeht, wird der 
Bewegungsbereich wieder auf 90 Grad erweitert. Mit den 4 vorhandenen Bewegungsmöglichkeiten am Arm ist eine ungehinderte Adduktion von ca. 25 Grad und eine Flexion von ca. 90 Grad möglich. Innen- und Außenrotation der Schulter sind bis ca. 70 Grad, ohne Beteiligung des Schultergürtels und damit ungehindert möglich (Tillmann, 2017; Palastanga et al., 2015). Für eine annähernd freie Beweglichkeit des Arms ist die Erweiterung des Mechanismus mit Teilbewegungen des Schultergürtels notwendig. Um die Komplexität des Gesamtmechanismus auf ein Minimum zu beschränken, werden nur die Freiheitsgrade des Sternoklavikulargelenks hinzugefügt. Bei vereinfachter Betrachtung entspricht es einem Kugelgelenk mit Drehzentrum im Kontaktpunkt zwischen Sternum und Clavicula (Schünke, 2014). Die Drehung um die Längsachse der Clavicula wird vernachlässigt. Daraus ergeben sich zwei weitere Einzelbewegungen, die als Kreuzgelenk im Mechanismus hinzugefügt werden und den Bewegungsbereich des Menschen auf das geforderte Ausmaß erweitern. Aufgrund der technischen Umsetzung erfolgt die Platzierung des Kreuzgelenks am Rücken und nicht, wie anatomisch vorgegeben, an der Brust.

\subsection{ANTRIEBSAUSLEGUNG}

Für den Antrieb des Exoskeletts ist ein Aktuator auszuwählen, der sowohl die Masse der Körpergliedmaßen als auch zusätzliche Massen in der Hand unterstützen kann. Der pneumatische Muskel besteht aus einer Elastomermembran in Schlauchform, die von einem Verstärkungsgeflecht umhüllt ist. Anschlussstücke an den Enden dienen dem Abdichten und dem Befüllen des Innenraums mit einem Fluid (Hesse, 2003). Der Aufbau und die Funktion des pneumatischen Muskels sind in Abbildung 4 dargestellt.

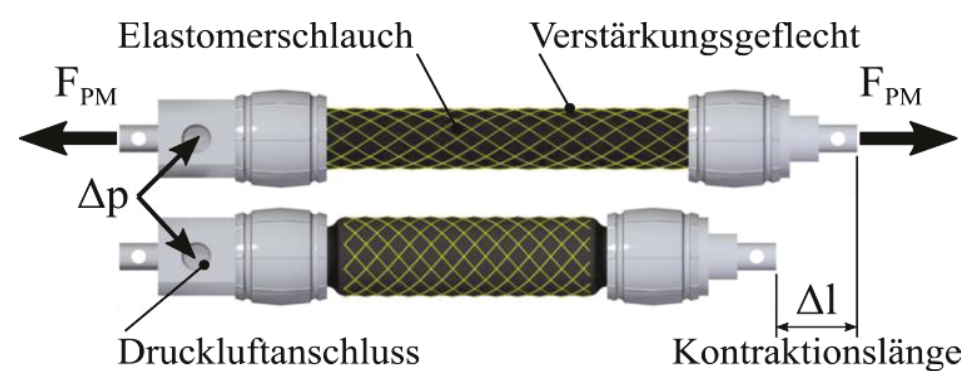

Abbildung 4: Aufbau pneumatischer Muskel in gestrecktem und kontrahiertem Zustand Quelle: $\quad$ Eigene Darstellung

Nach Hesse (2003) wird bei Erzeugung eines Innendrucks im Muskel eine Kontraktion hervorgerufen, die auf eine geometrische Kopplung zwischen dem Schlauch und der Verstärkungsfaser zurückzuführen ist. Die Funktionsweise resultiert aus dem Aufbau und ähnelt der des menschlichen Muskels. Durch Behinderung der Kontraktion entsteht eine Zugkraft $\left(\mathrm{F}_{\mathrm{PM}}\right)$ entlang des Muskels, die als Antrieb genutzt werden kann. Vorteilhaft ist das hohe spezifische Arbeitsvermögen, also die Erzeugung hoher Zugkräfte bei vergleichsweise 
geringem Eigengewicht, das dem Nutzer eine angemessene Kraftunterstützung liefern soll. Diese Kraft ist von der Kontraktionslänge abhängig und fällt zunehmend nichtlinear ab. Dieser Aspekt ist nachteilig für die Unterstützung eines Assistenzsystems, im industriellen Umfeld. Abbildung 5 zeigt den charakteristisch nichtlinearen Zusammenhang zwischen Muskelkraft, Kontraktion und Betriebsdruck. Beim Tragen eines Exoskeletts ist eine Unterstützung über den gesamten Bewegungsbereich und mit definierter Kraft notwendig. Pneumatische Muskeln können diese Anforderung nur erfüllen, wenn die Kontraktion begrenzt und ein Teil der verfügbaren Arbeit vernachlässigt wird. Die effiziente Ausnutzung der Muskelarbeit hängt dabei vom Lastpunkt ab. Er beschreibt die minimal erforderliche Kraft für die Unterstützung. Sowohl der Kraftabfall als auch die begrenzte Ausnutzung der Muskelarbeit sind entscheidende Nachteile.
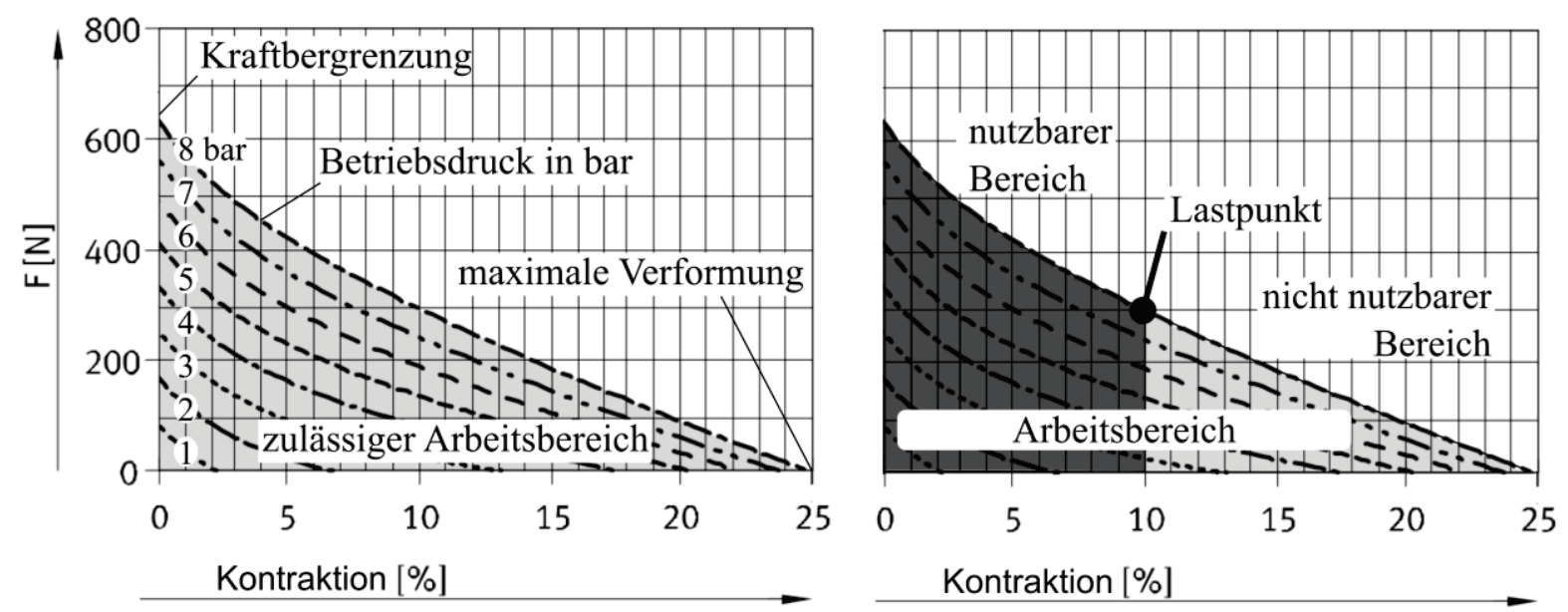

Abbildung 5: links: Kraft-Kontraktion-Diagramm eines PMA bei unterschiedlichen Betriebsdrücken, rechts: Verteilung der Muskelarbeit durch den Lastpunkt

Quelle: nach Hesse (2003)

Ein neuer Ansatz zur Eliminierung dieser Nachteile ist der Einsatz einer ungleichmäßigen Übersetzung mittels Bandgetriebe, wie bereits im Beitrag von Garbe (2017) vorgestellt. Hierbei wird ein, am pneumatischen Muskel befestigtes Seil über eine Seilscheibe mit variablen Radius umgelenkt, wodurch der Kraftverlauf bzw. das gesamte Arbeitsfeld gezielt verändert wird (Abbildung 6). Mit der Übersetzung kann eine variable, anwendungsspezifische Kraftkennlinie für unterschiedliche Anwendungsfälle erzeugt werden. 

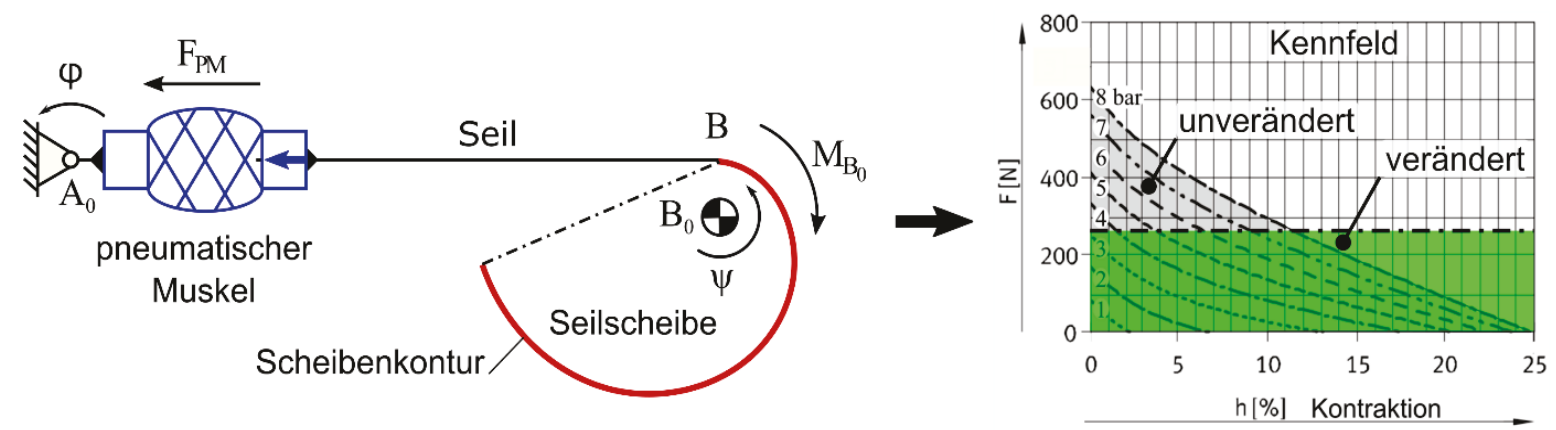

Abbildung 6: links: Getriebeschema ungleichmäßig übersetzendes Bandgetriebe, rechts: unverändertes und verändertes Kennfeld des pneumatischen Muskels

Quelle:

Eigene Darstellung

Die ungleichmäßige Übersetzung ist durch die Randbedingungen eingeschränkt, dass die entstehende Seilscheibe immer eine stetige und konvexe Kontur zum Drehpunkt besitzen muss. Erst damit ist eine definierte Übersetzung, durch tangentiale Auflage des Seils auf der Seilscheibenkontur gewährleistet. Bei der Verwendung einer einzelnen Seilscheibe für die Übersetzung ist nur eine Lösungsvariante der Seilscheibenkontur vorhanden. Diese Einschränkung kann dazu führen, dass die Seilscheibe, wegen ihrer Abmessungen für den mobilen Einsatz in einem Assistenzsystem, ungeeignet ist. Die Weiterführung dieser Entwicklung besteht in der Aufteilung der Gesamtübersetzung auf zwei seriell angeordnete Seilscheiben, mit der die Bauteilabmessungen reduziert werden können. Ausgehend vom pneumatischen Muskel erfolgt die Kraftleitung über ein Seil, auf die erste Seilscheibe. Mittels fester Verbindung zwischen den drehbar gelagerten Seilscheiben wird die Kraft auf das zweite Seil und von dort aus, an das aktuierte Gelenk übertragen. Festgelegte Parameter für die Auslegung der ersten Übersetzungsscheibe sind der Drehwinkel und die Kontraktionslänge des Muskels. Für die gezielte Veränderung der ersten Scheibenkontur wird die Gesamtübersetzung mit einer linearen Gleichung multipliziert, woraus die erste Teilübersetzung entsteht. Die zweite Teilübersetzung besitzt die qualitative Form der zuvor verwendeten linearen Gleichung. Bei der Auslegung stehen der kleinste und größte Radius der beiden Seilscheiben in direktem Zusammenhang. Dieser Aspekt ist bei der Auslegung hinsichtlich minimaler Biegeradien und maximaler Abmessungen zu berücksichtigen. Abbildung 7 zeigt den einfachen Aufbau des Mechanismus mit pneumatischen Muskel und zwei fest verbundenen Seilscheiben sowie den Seilen für die Kraftübertragung. 


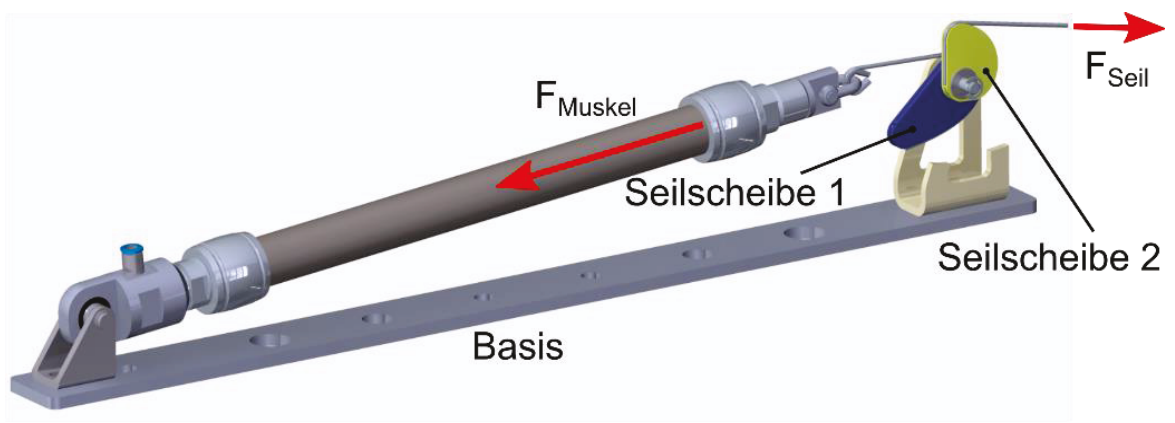

Abbildung 7: Antriebsmodul, Übersetzung eines pneumatischen Muskels mit zwei Seilscheiben Quelle: $\quad$ eigene Darstellung

Anwendungsbezogen sind die Modelle DMSP-10, -20 und -40 der Firma Festo für den Einsatz am Exoskelett relevant (Festo, 2017). Erreichbare Unterstützungsmomente für den gesamten Bewegungsumfang sind 3.65, 14.56 und 78.59 Nm, bei einem Betriebsdruck von 6 Bar. Der Aufbau kann als Antriebsmodul betrachtet und variabel am Assistenzsystem angebracht werden. Wegen der länglichen Form des Muskels bietet sich der Rücken als Ort für die Befestigung an. Abhängig von der Anzahl aktiv unterstützender Gelenke, kann die entsprechende Anzahl von Antriebmodulen nebeneinander oder in gestapelter Form zusammengefasst werden.

\section{SCHLUSSFOLGERUNG UND AUSBLICK}

Der vorgestellte Armmechanismus ist eine Entwicklung, die neben der theoretischen Auslegung im CAD Umfeld, auf einer Variantenstudie im Versuchsfeld aufbaut. Diese hat ergeben, dass die 6 Freiheitsgrade am Arm konstruktive Bewegungseinschränkungen der einzelnen Teilgelenke erfordern. Ohne diese sind unerwünschte Relativbewegungen die Folge, welche zu Abweichungen zwischen den menschlichen und technischen Gelenkachsen sowie singulären Stellungen führen. Des Weiteren benötigt das Exoskelett eine geeignete Mensch-Technik Schnittstelle. Über Anbindungen an den Armen müssen die auftretenden Kräfte, ohne übermäßigen Druck auf die Haut, definiert eingeleitet werden können. Wegen der notwendigen Länge der pneumatischen Muskeln ist eine Anbindung im Bereich des Rückens ratsam. Die Einleitung der Kraftunterstützung, auf das Schulter- und Ellenbogengelenk, kann platzsparend über Seile erfolgen. Seilhüllen leiten die Seilkraft von der Seilscheibe vom Antrieb zum Gelenk. Dort erfolgt die Umwandlung der Seilkraft, über eine Kreisscheibe, in ein Drehmoment. Voraussetzung ist die feste Anbindung der Seilhüllenenden an den jeweiligen Bauteilen. Der Wirkungsgrad bei der Kraftleitung wird maßgeblich von der Reibung im System und damit von der Biegung der Seilhülle bestimmt, weshalb diese zu minimieren ist. Für die Unterstützung eines Gelenks ist jeweils ein Antriebsmodul, mit einer, für die jeweilige Anwendung angepasste, Übersetzung vorgesehen. Es kann sowohl die Muskelgröße, als auch die Kraftkennlinie für jedes einzelne Gelenk angepasst werden. Der limitierende Faktor 
für die Größe und verwendete Anzahl der pneumatischen Muskeln ist die zur Verfügung stehende Rückenfläche und zusätzliche Masse. Muskelgrößen mit 10, 20 und $40 \mathrm{~mm}$ Innendurchmesser sind prinzipiell für die Anwendung relevant. Mit den zwei kleinen Varianten ist jedoch nur eine Teilentlastung der Gelenke möglich. Eine vollständige Entlastung, mit den im Beitrag genannten Zusatzmassen, erfordert die größte der drei Muskelvarianten. In weiterführenden Arbeiten ist zu untersuchen, welchen Einfluss die inhärente Nachgiebigkeit des pneumatischen Muskels auf die Krafteinleitung sowie Unterstützung hat und wie eine Energieversorgung mit mobilen Speichern gewährleistet werden kann.

\section{Danksagung}

Der Dank gilt allen kooperierenden Partnern im Projekt, „Entwicklung eines einfachen Exoskeletts zur Unterstützung von Hebe- und Montagetätigkeiten." Dieses Forschungsprojekt entstand im Rahmen des vom Bundesministerium für Wirtschaft und Energie geförderten ZIM (Zentrales Innovationsprogramm Mittelstand) AiF Kooperationsprojekts.

\section{LITERATURVERZEICHNIS}

Bundesanstalt für Arbeitsschutz und Arbeitsmedizin (2001). Leitmerkmalmethode zur Beurteilung von Heben, Halten, Tragen. Abgerufen von https://www.baua.de/DE/Themen/Arbeitsgestaltung-im-Betrieb/Physische-Belastung/ Leitmerkmalmethode/pdf/LMM-Heben-Halten-Tragen.pdf?_blob=publicationFile

Ebrahimi, A.; Gröninger, D.; Singer, R.; Schneider, U. (2017). Control Parameter Optimization of the Actively Powered Upper Body Exoskeleton Using Subjective Feedbacks. Stuttgart Exo-Jacket. In: 3rd International Conference on Control, Automation and Robotics, Japan. ISBN: 9781509060894

Festo (2017) Fluidic Muscle DMSP/MAS. Hg. v. Festo AG \& Co. KG. Abgerufen von https://www.festo.com/cat/de_de/data/doc_de/PDF/DE/DMSP-MAS_DE.PDF, zuletzt geprüft am 30.05.2018, 17:12Uhr.

Garbe, D.; Berger, M. (2017): Ungleichmäßige Übersetzung pneumatischer Muskeln mit Bandgetrieben. In: Michael Beitelschmidt (Hg.): 12. Kolloquium Getriebetechnik, Bd. 1. Dresden: S. 107-121.

Heine, A. (2010). MANIKIN. Möglichkeiten und Grenzen des Menschmodells. In: Maik Berger (Hg.): 2. Saxon Simulation Meeting und Mathcad Workshop. SAXSIM; Chemnitz: Univ.Verl. ISBN: 978-3-941003-12-5

Herr, H. (2009). Exoskeletons and orthoses: classification, design challenges and future directions. In: Journal of neuroengineering and rehabilitation 6. DOI: 10.1186/17430003-6-21.

Hesse, S. (2003). Der Fluidic Muscle. 150 Praxisbeispiele mit dem Pneumatischen Muskel. Hg. v. Festo AG \& Co. KG (Blue Digest on Automation).

Looze, M.; Bosch, T.; Krause, F.; Stadler, K. S.; O'Sullivan, L. W. (2016). Exoskeletons for industrial application and their potential effects on physical work load. In: Ergonomics (5), S. 671-681. 
Muramatsu, Y.; Kobayashi, H. (2014). Assessment of local muscle fatigue by NIRS development and evaluation of muscle suit -. In: Robomech J. (1), S. 1-11. DOI: 10.1186/s40648-014-0019-2

Palastanga, N. (2015). Soames, R.; Eggers, R. et al.: Anatomie und menschliche Bewegung. Strukturen und Funktionen. 1. Auflage auf Grundlage der 6. englischen Auflage. München: Elsevier Urban \& Fischer. ISBN: 978-3-437-45012-9.

Schünke, M. (2014). Topografie und Funktion des Bewegungssystems. Funktionelle Anatomie. 2. Aufl. s.I.: Georg Thieme Verlag KG. ISBN: 9783131185723.

Steinberg, U.; Windberg, H.-J. (2008). Heben und Tragen ohne Schaden. Bundesanstalt für Arbeitsschutz und Arbeitsmedizin. 5., unveränd. Aufl. Dortmund: Baua. ISBN: 978-388261-594-4.

Tillmann, B. (2017): Atlas der Anatomie des Menschen. Mit Muskeltabellen. Springer-Verlag $\mathrm{GmbH}$. 3., überarbeitete und erweiterte Auflage. Berlin, Heidelberg: Springer. ISBN: 978-3-662-49287-1. 